Boise State University

ScholarWorks

Psychological Sciences Faculty Publications and

Presentations

Department of Psychological Science

$11-1-2012$

\title{
The Role of Domain Satisfaction in Explaining the Paradoxical Association Between Life Satisfaction and Age
}

Kimberly K. McAdams

Boise State University

Richard E. Lucas

Michigan State University

M. Brent Donnellan

Michigan State University 
The Role of Domain Satisfaction in Explaining the Paradoxical Association between

Life Satisfaction and Age

\author{
Kimberly K. McAdams \\ Richard E. Lucas \\ M. Brent Donnellan \\ Michigan State University
}

Draft Date: January 19, 2010

Word Count: 3,136 


\begin{abstract}
Although aging is associated with declines in many life circumstances, overall life satisfaction does not appear to sharply decline with age. One explanation for this paradoxical finding is that several life domains improve with age such that increases in certain domains balance the decreases in others. Life and domain satisfaction data from eight years of the British Household Panel Study were analyzed to evaluate this hypothesis. Results showed that although domain satisfaction scores demonstrate distinct trajectories, the aggregate of these distinct trajectories resembled the overall life satisfaction trajectory. These findings have implications for top-down and bottom-up models of life satisfaction.
\end{abstract}

Word Count: 100

Key Words: Life satisfaction, domain satisfaction, British Household Panel Study, bottom-up vs. top-down models

Main Text Word Count: 2,521 
As people age, they often experience declining life circumstances, such as a poorer health and fewer social relationships (Baltes \& Mayer, 1999). However, in spite of these negative circumstances, overall life satisfaction, or the cognitive assessment of one's life (Lucas, Diener, \& Suh, 1996) does not show such a pronounced decline with increasing age. For instance, Blanchflower and Oswald (2008) used cross-sectional data from multiple nations to demonstrate that life satisfaction follows a curvilinear pattern that reaches its lowest point at middle age but then increases into later adulthood. Baird, Lucas, and Donnellan (2009) extended these analyses using two very large household panel studies, and showed that the life satisfaction generally does not decline until after age 70 . This trajectory is interesting given that health usually declines during this age period, yet overall life satisfaction continues to increase (Baird et al., 2009). In this paper, we evaluate the plausibility of various explanations for this seemingly paradoxical trend whereby overall life satisfaction appears to remain high even as many life circumstances deteriorate.

There are at least two explanations for the paradoxical findings regarding the trajectory of life satisfaction across the lifespan. First, it is possible that life satisfaction may not be sensitive to changes in one's circumstances. Instead, some people may be inclined to see the world through "rose-colored glasses" regardless of what is occurring in their everyday lives. In contrast, other people may be likely to put a negative spin on everything, even if their lives are going well. According to this view, life satisfaction depends more on an individual's temperamental dispositions than on her or his current life situation. Given that age-linked trends for Extraversion and Emotional Stability (the strongest personality correlates of life satisfaction; see Romero, Villar, Luengo, \& Gómez-Fraguela, 2009) are generally in opposite directions 
(Lucas \& Donnellan, 2009), the net consequence might be a fair degree of absolute stability in life satisfaction judgments.

Alternatively, it is possible that some life domains may change in positive directions with age, which would counteract negative changes that occur in other domains. In other words, it is possible that the domain-specific judgments change in systematic ways with age but that the increases and decreases tend to average out over the course of the lifespan. Socio-emotional selectivity theory, in particular, predicts that individuals become more selective about how they invest their time and psychological energy as they approach the end of life. This increased selectivity could result in corresponding increases in satisfaction with life domains that involve social relationships and leisure activity.

Currently, there are two main theories that explain how domain-specific judgments of life satisfaction are related to overall life satisfaction: top-down and bottom-up theories. Top-down models specify that one's overall level of life satisfaction influences his or her perception of the specific domains of life. Another way of conceptualizing a top-down model is to think of the "rose-colored glasses" metaphor. Someone who sees the world through rose-colored glasses always looks for a positive way to interpret the situation, regardless of how objectively negative it is. In other words, a person who is temperamentally happy might interpret the various domains in life as being quite positive, even if they were objectively negative. Importantly, if top-down processes drive the association between global life satisfaction and domain satisfactions, then domain satisfaction scores should change in similar ways over the course of the lifespan-as a person's global satisfaction changes, the top-down influence should lead to corresponding changes in domain satisfaction ratings. 
In contrast to the top-down model, the bottom-up model posits that an individual evaluates different life domains separately, and that these specific evaluations influence one's overall life satisfaction. In other words, overall life satisfaction can be conceptualized as the aggregate of multiple domain satisfaction ratings (Diener, 1984; Hsieh, 2003). Importantly, if the bottom-up model is correct, then the trajectories for the individual domains may be quite different than the trajectory for the global judgment (see Diener, 1984). However, if life satisfaction does follow a bottom-up model, we should see that the trajectory for the aggregate of the important domains in a person's life matches the global life satisfaction trajectory.

All in all, an investigation of domain-specific judgments of life satisfaction across the lifespan could contribute important data for two ongoing controversies in the study of life satisfaction-why the trajectory for overall life satisfaction follows a particular pattern and whether the top-down or bottom-up model is more plausible. In particular, we evaluate trajectories of domain-specific satisfaction judgments in eight areas of life and estimate how these ratings are related to the overall life satisfaction trajectory. The current study is unique in that it uses longitudinal data from a nationally representative panel study to assess these agerelated trajectories. Indeed, this study will provide further insight into the factors that underlie the paradoxical link between age and life satisfaction that has been demonstrated in past research.

\section{Method}

\section{Participants}

Participants for this investigation were obtained from Waves F (1996) through N (2004) of the British Household Panel Study (BHPS; University of Essex, Institute for Social and Economic Research, 2006), which is a panel study of British households that started in 1991. 
Data was not obtained for Waves A through E or for Wave K (2001) because satisfaction measures were not collected during these waves of the BHPS. Participants were selected using multi-stage probability design with systematic sampling, and the sample is designed to be representative of the adult population of the United Kingdom. The sample was restricted to participants between the ages of 16 and 93 , yielding a total of 24,094 participants $(53.6 \%$ women; $M$ age $=42.59$ years old). Because only participants who were working answered the question about job satisfaction and only participants who were in a committed relationship were asked questions about relationship satisfaction, the sample was restricted further for these questions.

Measures

Both life and domain satisfaction ratings were assessed using the stem: "How dissatisfied or satisfied are you with..." Life satisfaction was assessed with the item "your life overall." The eight domain satisfaction questions asked about participants' health, income of household, house/flat, spouse/partner, job, social life, amount of leisure time, and use of leisure time. Participants rated each item on a scale from 1 ("not satisfied at all") to 7 ("completely satisfied"). Means, standard deviations, and the average year-to-year retest reliability for each item are reported in Table 1.

\section{Results}

We used multilevel modeling techniques to estimate age-related trajectories for overall life satisfaction, each domain satisfaction question, and the aggregate of all eight domain satisfaction questions. Age (centered around the sample grand mean), along with agepolynomials up to the fourth power were entered into a multilevel model predicting each 
outcome variable. For the sake of brevity, detailed results are not presented, though they are available from the authors on request.

If the top-down model is correct, each domain satisfaction rating is primarily influenced by overall life satisfaction, and thus should show similar trajectories over the course of the lifespan. Figure 1 shows that this prediction is not supported by the data. Panel A shows that the trajectories diverge considerably across the eight life domains.

In accordance with expectations, health satisfaction declines relatively steadily over the course of the lifespan. However, other domains show distinct patterns. Job satisfaction ${ }^{1}$ and income satisfaction trajectories are relatively flat throughout much of the lifespan, but then satisfaction in these domains increases somewhat dramatically (reflecting an increase by approximately .45 standard deviations for job satisfaction and an increase of approximately .30 standard deviation for income satisfaction) in later life. Relationship satisfaction increases sharply from the teens to the twenties, remains somewhat flat until the mid-forties, and then increases steadily until the late seventies. Satisfaction with social life, amount of leisure time, and the use of one's leisure time all decrease quite sharply from the late teens to the early forties (presumably as a person's career increases in importance), but then increase quite rapidly from the mid-forties until the late seventies. Satisfaction with housing shows a similar pattern, though the initial drop is not quite as extreme.

Panel B of Figure 1 plots the age-related trajectory for global life satisfaction (which was previously reported in Baird et al., 2009) along with the trajectory for an aggregated domain satisfaction measure, which is the simple average across all eight domain satisfaction ratings. These results show that when all domain satisfaction ratings are aggregated, the overall trajectory

\footnotetext{
${ }^{1}$ The job satisfaction trajectory ends at 75 because this is the last age for which we had sufficient data to include in the analysis. Participants only reported on job satisfaction if they currently held a job, which was uncommon for individuals past retirement age.
} 
is remarkably similar to that of the global life satisfaction scale, even though the trajectories for the individual components diverge quite a bit. For both the global scale and the aggregate measure, satisfaction scores are predicted to drop from approximately 5.4 among late teens to about 4.9 in the late thirties or early forties, and then to increase to about 5.5 in the mid seventies. After this point, both the global and aggregated measures are predicted to drop among the oldest participants.

\section{Discussion}

Much controversy surrounds the connection between objective life circumstances and life satisfaction judgments. Although early theories generated this prediction that associations between life circumstances and satisfaction judgments would be strong, much existing research shows that the actual effects are often surprisingly small (Diener, Suh, Lucas, \& Smith, 1999). Some have interpreted these associations to mean that people do not construct judgments in a bottom-up fashion. Instead, it may be that temperamental processes drive life satisfaction judgments, and that dispositionally happy people will report being satisfied with their life and the domains in it, even if those domains are objectively unpleasant and undesirable. The association between age and life satisfaction plays a role in these debates because many objective life circumstances are known to systematically decline with age. Thus, the fact that life satisfaction does not decline with age, at first glance, provides further support for the idea that actual life circumstances play only a small role in life satisfaction judgments.

The goal of the current study was to take a different approach to examining the association between age and life satisfaction than has been used in the past. Specifically, we investigated the alternative possibility that global life satisfaction does not decline simply because certain domains in life improve with age, and these improvements balance the more 
salient declines that are often the focus of research attention. Results confirmed that age-related trajectories varied considerably for satisfaction with different important life domains. Consistent with expectations, health satisfaction declined steadily across the lifespan. Satisfaction with work and income were relatively flat in early to mid adulthood before increasing in late adulthood. Domains involving social and leisure activities declined quite dramatically in early adulthood and then rebounded in late adulthood. Importantly, however, once the distinct domains were simply averaged, the trajectory for the aggregate measure closely mirrored the trajectory for global life satisfaction judgments.

Although this study does not directly test the processes by which life satisfaction judgments are constructed, it provides support for a bottom-up model. If the alternative top-down models are correct, global feelings of life satisfaction influence judgments of narrow domainshappy people see the world through rose-colored glasses that color their interpretation of the objective events in it. Such models would lead to the prediction that domain satisfaction ratings should change in similar ways over the lifespan and that these trajectories should mirror the changes in overall life satisfaction. The current results point to the conclusion that the trajectories for different domains in fact differ. It is only when these distinct domains are aggregated that a pattern similar to that for global life satisfaction emerges. This pattern is consistent with the idea that people are constructing these judgments in a bottom-up fashion and that these domains are more or less equally weighted.

This study contributes to existing work by moving researchers closer to an explanation of the age-related changes that occur in global life satisfaction. As it stands, existing research supports the curvilinear effect first identified by Oswald and Blanchflower (though the effect does not always emerge and it may be moderated by certain characteristics of the nation; Deaton, 
2008). The remaining questions are now concerned with an explanation for this seemingly robust trend. By examining variables like domain satisfaction, the processes that underlie this global effect can be understood more precisely. The current results suggest that different domains change in different ways over the course of the lifespan, and the positive changes in income, jobs, and social life can compensate for declines in health that people experience in later life. Of course, our investigation assessed domain satisfactions, so future research should objectively examine these life domains.

Although the present results are compelling, there are several additional issues that warrant future study. First, it is necessary for future research to examine individual differences in association with changes in life satisfaction. Previous research has found that the traits of Neuroticism and Extraversion moderate the degree to which both negative and positive affect change with age (Charles, Reynolds, \& Gatz, 2001). Therefore, it is also possible that Neuroticism and Extraversion may moderate changes in each of the life satisfaction domains. Additionally, it is necessary for future researchers to investigate if change in life domain satisfaction is an individual difference. Because previous findings have illustrated that individuals differ in the degree to which their Neuroticism and Extraversion scores change over time (Mroczek \& Spiro, 2003), it is possible that individuals may also vary regarding to what extent their life satisfaction scores change with age.

On a final note, it is worth pointing out that the initial impression that life circumstances decline with age is supported when the focus is restricted to the oldest members of the sample. Notably, the oldest old are often excluded from studies of life satisfaction, and thus the trajectories that are typically estimated may not capture the decline in these later years. With the exception of job satisfaction (which may present a misleading picture because retirees are not 
asked this question) and income satisfaction, all of the domains that we investigated - and the overall life satisfaction rating - showed fairly substantial declines after age 80 . This finding provides some support for the idea that life satisfaction declines when objective life circumstances decline; it may just be that the most salient declines do not occur until the very end of life.

In summary, life satisfaction has been shown to have a curvilinear trajectory across the lifespan, and this trajectory makes sense in light of the changes that occur in the underlying domain satisfaction ratings. The changes that occur are not trivial—seventy-year-old respondents reported overall life satisfaction scores that were approximately .40 standard deviations above that of forty-year olds. Although some domains decline with age, these appear to be balanced by increases in other domains, at least until the very end of life. In short, domain specific judgments appear to wax and wane with age, but these appear to balance out over the course of the lifespan. 


\section{References}

Baird, B., Lucas, R. E., \& Donnellan, M. B. (2009). Life Satisfaction across the lifespan: Evidence from two nationally representative panel studies. Manuscript under review.

Baltes, P.B., \& Mayer, K.U. (1999). The Berlin Aging Study: Aging from 70 to 100. Cambridge, United Kingdom: Cambridge University Press.

Blanchflower, D. G., \& Oswald, A. J. (2008). Is well-being U-shaped over the life cycle? Social Science \& Medicine, 66, 1733-1749.

Charles, S. T., Reynolds, C. A., \& Gatz, M. (2001). Age-related differences and change in positive and negative affect over 23 years. Journal of Personality and Social Psychology, 80, 136-151.

Deaton, A. (2008). Income, health and wellbeing around the world: Evidence from the Gallup World Poll. The Journal of Economic Perspectives, 22, 53-72.

Diener, E. (1984). Subjective well-being. Psychological Bulletin, 95, 542-575.

Diener, E., Suh, E. M., Lucas, R. E., \& Smith, H. L. (1999). Subjective well-being: Three decades of progress. Psychological Bulletin, 125, 276-302.

Hsieh, C. M. (2003). Counting importance: The case of life satisfaction and relative domain importance. Social Indicators Research, 61, 227-240.

Lucas, R. E., Diener, E., \& Suh (1996). Discriminant validity of well-being measures. Journal of Personality and Social Psychology, 3, 616-628.

Lucas, R. E., \& Donnellan, M. B. (2009). Age differences in personality: Evidence from a nationally representative Australian sample. Developmental Psychology, 45, 1353-1363.

Mroczek, D. K., \& Spiro, A., III (2003). Modeling intraindividual change in personality 
traits: Findings from the normative aging study. Journal of Gerontology, 58B, $153-165$.

Romero, E., Villar, P., Luengo, M. A., \& Gómez-Fraguela, J. A. (2009). Traits, personal strivings and well-being. Journal of Research in Personality, 43, 535-546.

University of Essex, Institute for Social and Economic Research (2006). British Household

Panel Survey; Waves 1-14, 1991-2005 [computer file]. 2nd Edition. Colchester, Essex: UK Data Archive [distributor]. SN: 5151. 


\begin{abstract}
Author Note
Correspondence regarding this manuscript should be addressed to Kimberly K. McAdams, Department of Psychology, Michigan State University, East Lansing, MI 488233. Email:

assadkim@msu.edu. The BHPS data were originally collected by the ESRC Research Centre on Micro-social Change at the University of Essex, now incorporated within the Institute for Social and Economic Research. Neither the original collectors of the data nor the Archive bear any responsibility for the analyses or interpretations presented here. Preparation of this manuscript was supported by National Institute on Aging grants 1R03AG026028-01 and 1R03AG02874401.
\end{abstract}


Table 1

Means, Standard Deviations, and Average Year-to-Year Retest Reliability

\begin{tabular}{llll}
\hline$\underline{\text { Variable }}$ & $\underline{M}$ & $\underline{S D}$ & $\underline{r}$ \\
Health & 4.98 & 1.63 & .59 \\
Income & 4.56 & 1.63 & .59 \\
House/Flat & 5.42 & 1.47 & .52 \\
Spouse/ & 6.23 & 1.22 & .55 \\
Partner & & & \\
Job & 5.01 & 1.46 & .48 \\
Social Life & 4.98 & 1.52 & .59 \\
Amount of & 4.84 & 1.65 & .58 \\
Leisure Time & & & \\
Use of Leisure & 4.92 & 1.56 & .56 \\
Time & & & \\
Overall Life & 5.24 & 1.31 & .57 \\
Satisfaction & & & \\
\hline
\end{tabular}




\section{Satisfaction Figure Caption}

Figure 1: Domain satisfaction and overall life satisfaction trajectories. 

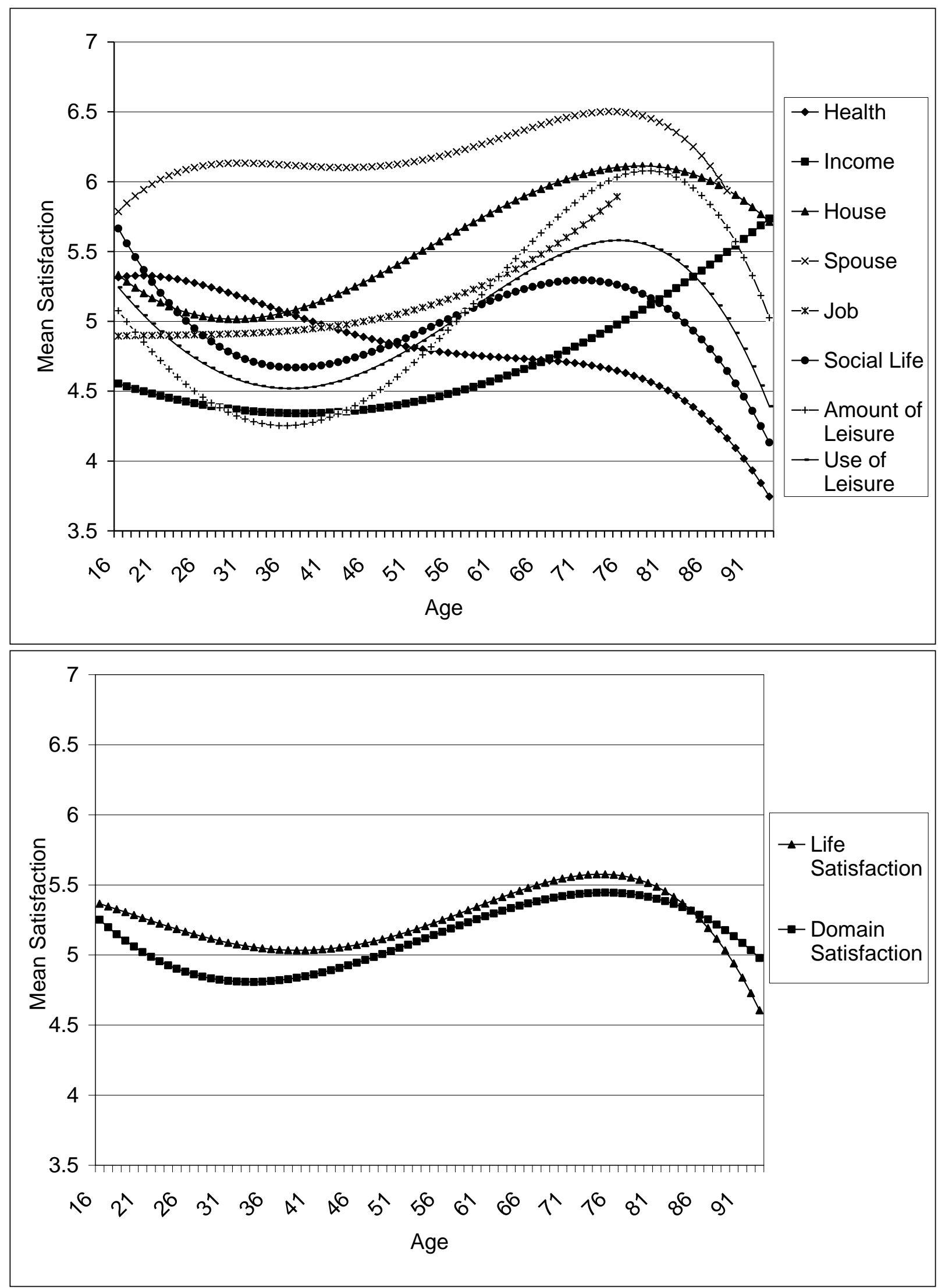